\title{
The Influence of Magnetic Field on the Enrichment by Polymeric Membranes
}

\author{
Z. J. Grzywna ${ }^{1}$, A. Rybak ${ }^{2 *}$, P. Borys $^{3}$, K. Pawelek ${ }^{4}$ \\ ${ }^{1,2,3 \& 4}$ Department of Physical Chemistry \& Technology of Polymers, Section of Chemical Physics and Biophysics, \\ Faculty of Chemistry, Silesian University of Technology, Ks. Marcina Strzody 9, 44-100 Gliwice, Poland
}

\begin{abstract}
An idea of using magnetic field to enhance oxygen transport, as an effective factor for the air enrichment, has already been successfully demonstrated. Direct consequence of this concept was to produce polymeric magnetic membranes by casting technique. An analysis of experimental data, obtained from transient and stationary states of mass transport, allowed to formulate a hypothesis of a "dead thickness" of magnetic membranes in question, i.e. the bulk polymer phase as a part of membrane with no magnetic channels but limiting the overall transport through it. It will be shown that removing this part increases the rate of transport and enhances the air separation. To complete the overall approach, the theory is provided both on phenomenological and molecular levels. The simulation by Monte Carlo and Brownian dynamics, to show some molecular properties of magnetic field and its activity during separation, was performed.
\end{abstract}

Keywords: Air separation, diffusive transport, magnetic membranes, Monte Carlo modelling, dead thickness

\subsection{INTRODUCTION}

An importance of air enrichment in oxygen or final air separation to pure oxygen and nitrogen contaminated by noble gases, carbon dioxide, water vapour, etc. is unquestionable. The list of potential users of an enriched air (to say nothing about pure oxygen) is rather long, ranging from hospitals, steel factory and biotechnology plants to regular habitants of large cities with polluted air. The crucial factor in handling this problem is the cost. With no detailed analysis, one can say that membrane separation is unbeatable on this respect as compared with cryogenic, and swing adsorption methods. Among the membrane techniques, there is however still a large variability. The membranes working as molecular sieves [5] can be pretty expensive while magnetic membranes [4], are much, much cheaper.

\footnotetext{
* Corresponding to:A. Rybak (email: Aleksandra.Rybak@polsl.pl)
}

It should be emphasized that gas separation is just one of the two most important membrane processes. The other one, the liquid separation is even more important for it covers the water purification, distillation, etc. $[6,7,8]$. The high quality water is one of the crucial importance in a global sense both for social, and industrial reasons $[9,10]$.

The concept of air enrichment by magnetic membranes, i.e. the polymer membranes (matrix) with dispersed magnetic powder finally magnetized up to $1.75 \mathrm{mT}$ has been introduced only recently $[1,2,3,4]$.

Although the idea is promising it definitely needs further improvements. In this paper, we will present a logical "feedback" between the theory, modelling and experiments. Special emphasis is put on the granulation of magnetic powder, which has a direct connection with the magnetic channels size and also on the "dead length" of magnetic membrane, i.e. the part of membrane with no magnetic channels. 


\subsection{METHODS}

\subsection{Membranes' Casting}

The flat ethylcellulose (EC) and poly (2,6-dimethyl1,4-phenylene oxide) (PPO) membranes of thickness 20-80 $\mu \mathrm{m}, \mathrm{EC}$ and PPO magnetic membranes of thickness 90-370 $\mu$ m (depended on magnetic powder granulation, and on the amount of added powder), were obtained using casting method. Membranes were cast from 3\% EC solution in 40:60 ethanol/toluene or $2.5 \% \mathrm{PPO}$ solution in trichloroethylene (TCE).

Magnetic membranes were made by pouring the EC or PPO solution with a dispersed neodymium magnetic powder (of the appropriate amount: $1.3-1.8 \mathrm{~g}$ and granulation: $20-32 \mu \mathrm{m}$ ), into a Petri dish and then evaporating the casting solvents in the external field of a coil (stable magnetic field with the range of induction 0-40 $\mathrm{mT}$ ) for $24 \mathrm{~h}$. Obtained membranes were removed from Petri dish (with some distilled water) and dried in $40^{\circ} \mathrm{C}$ for at least two days before any analysis. For membranes with dispersed magnetic powder, the permeation measurements were carried out, before (just powder) and after magnetization (magnetic membranes). These membranes were magnetized in the magnet field with magnetic induction $B$ approximately of $2.5 \mathrm{~T}$. Teslameter FH 54 was used for measurement of membrane magnetic induction. The membranes were stored in an exsiccator under the vacuum conditions $(p=3 \mathrm{~mm} \mathrm{Hg})$.

\subsection{Permeation Set Up}

Experimental setup APG-1 (Figure 1), described in paper [2] was used for measurement of the nitrogen, oxygen and air permeability. This setup was furnished with a gas chromatograph HP $5890 \mathrm{~A}$, which let us to measure the oxygen and nitrogen concentration in permeate. The main part of this experimental setup was a diffusive chamber, where the membrane in the form of a disc was placed in. The membrane effective area was 19.63 $\mathrm{cm}^{2}$. This setup was used for a low-pressure (from 0.1 to $1.0 \mathrm{MPa}$ ) analysis of gas permeation. After installation of membrane in the diffusive chamber, the suitable pressure difference was established

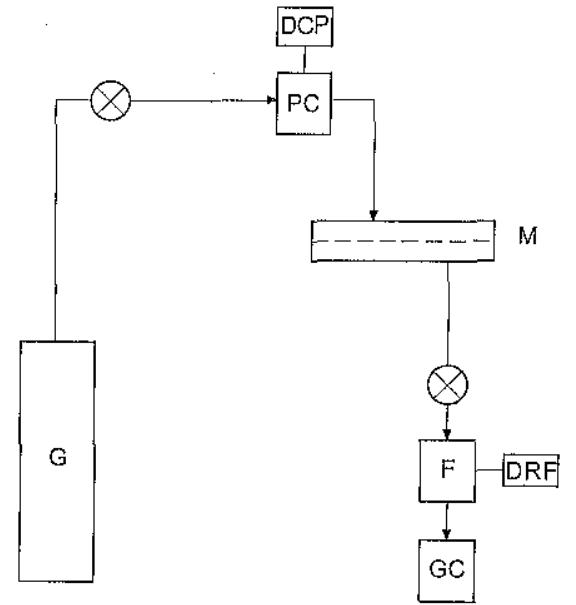

Figure 1 Scheme of the experimental setup APG-1, G-gas cylinder, F - flowmeter, DRF - digital registrar of flowmeter, PC - pressure controller, DCP - digital controller of pressure, $\mathrm{M}$ - diffusive chamber, GC - gas chromatograph.

(0.3-0.7 $\mathrm{MPa}$, depending on membrane) and controlled by an electronic pressure meter and controller EL-press P-506C.

After that, the flow rate of permeate was recorded using Flow-Bus flowmeter (with a range 0-3 $\mathrm{ml} / \mathrm{min}$ ) with a computer data acquisition. All measurements were carried out in room temperature. The Time Lag method and D1-D8 system were used for analysis of experimental data $[2,4]$. Few important diffusion and permeation coefficients, like: $D_{L}, \bar{D}, P$ were obtained using Time Lag method, while $D_{3}, D_{4}, D_{5}$ coefficients were obtained by D1-D8 system [2, 4]. Experimental permeation data for the oxygen and nitrogen, both for individual, pure gases, as well as components of air were collected to analyse the system, i.e. membrane and permeating gas, using appropriate formulas [2] supported by the percentage of air enrichment.

\subsection{THEORY}

\subsection{Mathematical Description (Modelling)}

Since we postulate that magnetic membranes have the porous structure, the porous medium equation 
should be the one to describe the transport through them. Porous medium equation (PME) is the nonlinear, parabolic partial differential equation (PDE) that is supposed to describe the transport of gases and liquids through the porous medium (membrane) as well as thermal propagation in plasma [11].

To derive the PME we start with an equation of state [12]

$$
p(x, t)=p_{o} \rho^{\alpha}(x, t), \alpha \in[1, \infty]
$$

supplemented by the mass conservation

$$
\kappa \frac{\partial \rho}{\partial t}+\operatorname{div}(\pi \vec{V})=0
$$

and Darcy's law

$$
v \vec{V}=\mu \nabla p
$$

where: $p_{o}, \alpha$-constant, $\vec{V}$ - velocity, $v$ - viscosity, $\mu$ - permeability, $p(x, t)$ - pressure, $\rho(x, t)$ - density, $\kappa$ - the porosity (it specifies what amount of mass with specified velocity actually gets through the pore in the mass balance equation).

Eliminating $p$ and $\vec{V}$ from the above (eqs. 1-3), we get

$$
\frac{\partial \rho}{\partial t}=\frac{\alpha p_{0} u}{v_{K}(1+\alpha)} \operatorname{div}\left[m \rho^{m-1} \nabla \rho\right]
$$

If we put $D_{o}=\frac{\alpha p_{o} u}{v \kappa(1+\alpha)}, f(\rho)=m \rho^{m-1}$ and $m=1+\alpha$, we get

$$
\frac{\partial \rho}{\partial t}=\operatorname{div}[D(\rho) \operatorname{grad} \rho]
$$

with

$$
D(\rho)=D_{o} f(\rho)
$$

or

$$
\frac{\partial \rho}{\partial t}=D_{o} \Delta \rho^{m}
$$

For the Clapeyron gas, $m=2$.

To meet expectations of the present experimental data we have modeled our system by the set of two, weakly coupled, position and induction dependent differential equations of the form [13]:

$$
\frac{\partial C_{O_{2}}}{\partial t}=\frac{\partial}{\partial t}\left[D_{O_{2}}(B, x) \frac{\partial C_{O_{2}}}{\partial x}\right]
$$

$$
\frac{\partial C_{N_{2}}}{\partial t}=\frac{\partial}{\partial t}\left[D_{N_{2}}(B, x) \frac{\partial C_{N_{2}}}{\partial x}\right]
$$

where

$$
\begin{aligned}
& D_{O_{2}}(B, x)=D_{O_{2 P}}(1-420 B)+D_{c h} \exp \left[2 P_{a g r}\right. \\
& \left.+1\left(1-P_{a g r}\right)\right] \cdot \exp \left\{-\left(\frac{L-x}{W_{O}+W_{B} \sqrt{B}}\right)^{2}\right\}
\end{aligned}
$$

and

$D_{N_{2}}(B, x)=D_{N_{2, p}}(1-420 B)+D_{c h} P_{a g r} \frac{D_{O_{2,0}}}{D_{N_{2,0}}}$

$\exp (2) \cdot \exp \left\{-\left(\frac{L-x}{W_{O}+W_{B} \sqrt{B}}\right)^{2}\right\}$

Where:

$D_{O_{2 P}}$ - diffusion coefficient of the oxygen in the polymer phase,

$D_{N_{2 P}}$ - diffusion coefficient of the nitrogen in the polymer phase,

$D_{c h}$ - magnetic channel diffusion coefficient,

$P_{\text {agr }}$ - aggregation probability between oxygen and nitrogen,

$L \quad$ - dead length of the magnetic membrane with no magnetic particles,

$W_{0}$ - average length of travel within a magnetic channel

$W_{B}$ - represents the effect of membrane magnetization (B) on length of travel within a magnetic channel,

$C_{O_{2,0}}$ and $C_{N_{2,0}}$ represent the feed concentrations of oxygen and nitrogen, respectively.

Please, observe that mathematical modeling is not unique i.e. there can be two or more formal descriptions of one given problem.

\subsection{Molecular Modelling}

The simulation was based on Monte-Carlo methods addressed to random walk of oxygen and nitrogen molecules, respectively. The diffusion space was modeled (schematically presented on Figure 2 ) as $3 \mathrm{D}$ lattice with $100^{*} 100 * 500$ nodes, with constant node displacement $d x=0.2 \mu \mathrm{m}$ which corresponds to physical dimensions of simulated membrane equal to $20 \times 20 \times 100 \mu \mathrm{m}$ (cross sectional area 
limited due to computational effort), assuming the average diameter of neodymium powder varying from $100 \mathrm{~nm}$ to $30 \mu \mathrm{m}$. The structure shown in Figure 2 is only a part of the magnetic membrane including one abstract percolation cluster (created for the modelling), which is a system of interconnected magnetic particles. The distribution of particles of magnetic neodymium powder (of given size) in the lattice was used as determined in another simulation based on random walk of neodymium particles in external magnetic and gravitational fields. The nodes adjacent to these particles were assumed as a region of magnetic channels. The net magnetic field in all nodes was determined. Finally, a random walk of paramagnetic oxygen and diamagnetic nitrogen in the presence of a magnetic field was performed on the lattice.

The initial and boundary conditions were as follows:

$C(x, y, z, t=0)=0, C(x, y, z=0 \mu \mathrm{m}, t)=$ const, $C(x, y, z=100 \mu \mathrm{m}, t)=0$

The remaining boundary conditions were of reflecting type.

The probabilities for entering of oxygen molecules to the channels or to stay in the polymeric region were said to be proportional to Boltzmann factors of the form:

$$
B_{f} \approx \exp \left(\frac{-\Delta U}{k T}\right)
$$

where $\Delta U$ is a change of potential field values between channel and polymeric region in appropriate position, $k$ is the Boltzmann constant and $T$ is the temperature. For diamagnetic nitrogen the changes in potential energy are negligible compared to thermal energy, so these probabilities are assumed to be equal [13]. The oxygen in turn becomes attracted due to the magnetic Weiss field interaction energy.

The diffusion coefficient in polymeric membrane is approximately equal to $D=10^{-7}$ $\left[\mathrm{cm}^{2} / \mathrm{s}\right]$. The Knudsen diffusion coefficient for a channel of radius $r[\mathrm{~m}]$ can be determined from the formula [14].

$$
D_{K}=-r \sqrt{\frac{8 R T}{\pi M}}
$$

where $R$ is a gas constant $[J /(\operatorname{mol} K)], T$ is a absolute temperature $[K]$ and $M$ is molar mass $[\mathrm{kg} / \mathrm{mol}]$. The value of Knudsen diffusion coefficient, even for very narrow channels, (nanometers in size) is at least few orders of magnitude greater as compared to the normal diffusion coefficient in polymer membrane. The polymer membranes are dense. But in the case of magnetic membranes we suspect that at the border between magnetic particle and a polymer appear some pores. We do not know their size, but we suspect that it is no less than the Van der Vaals diameter of the oxygen, nitrogen or their aggregate.

So, when a diffusing molecule is found to be in the channel region it undergoes random walk in it, but it performs at least few jumps within the time needed for one jump in polymeric region (since $D=\delta^{2} / \tau, \delta$ fixed). The ratio is assumed to be grater for oxygen which "sticks" to the magnetic neodymium surface better than the nitrogen.

After performing the desired number of jumps molecules can go back to the polymeric region or stay in the channel. The probabilities are determined in similar ways as described previously. The channel is open on the permeation side of the membrane, so eventually molecules can also leave the membrane directly from the channel.

As a result of simulations fluxes of oxygen and nitrogen were obtained as a function of applied magnetic field and neodymium granulation.

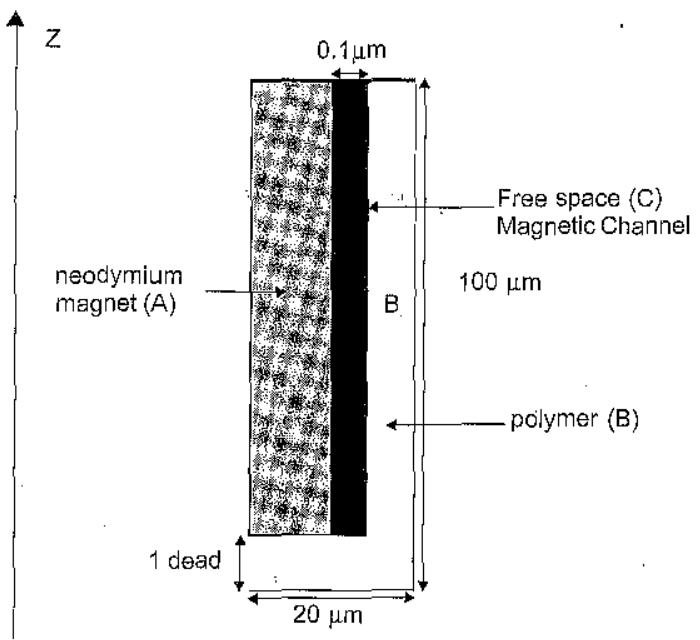

Figure 2 The schematic 2D representation of a model 


\subsection{RESULTS AND DISCUSSION}

So far, accumulated experimental data are collected in Table 1 and 2, just for one size of magnetic neodymium (actually the Nd-Fe-Nb-B alloy) of about $20-32 \mu \mathrm{m}$ in average.

The maximum air enrichment, for this granulation, was about $60 \%$ (Figure 3 ).

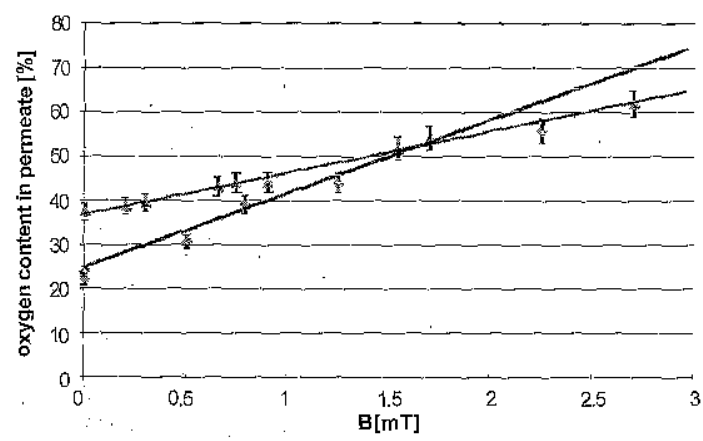

Figure 3 Dependence of the oxygen content in permeate vs. magnetic field induction. Points marked as rhombus were obtained in experiment for EC matrix, points marked as triangles were obtained in experiment for PPO matrix. Straight lines were predicted by the theory

The limit of about $60 \%$ of air enrichment could be overcome by decreasing the magnetic powder granulation that is clearly shown by molecular modelling (Figure 4).

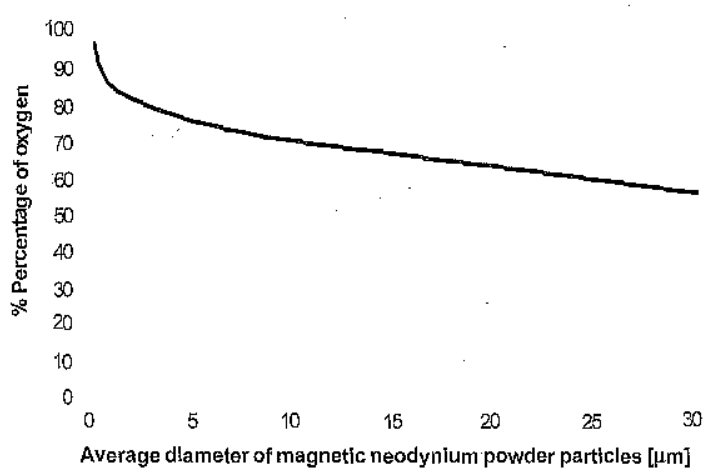

Figure 4 Plot of the oxygen concentration in the enriched air as a function of average diameter of the neodymium powder particles. Simulation was performed for diameters ranging from $0.1 \mu \mathrm{m}$ to $30 \mu \mathrm{m}$
The above results support an idea of magnetic channels created in the neighbourhood of magnetic powder percolation clusters (c.f. Figure 5).

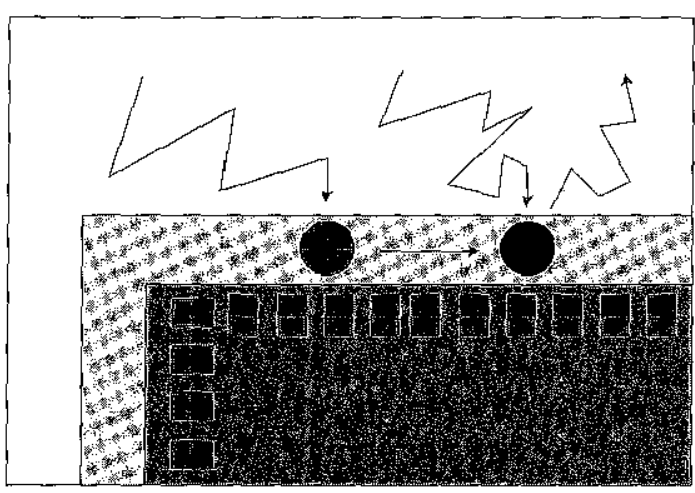

Figure 5 An (over) simplified illustration of the magnetic channel operation. Paramagnetic oxygen (a red-blue magnetic dipole) is latched within the channel, while diamagnetic nitrogen (brown particle) is not

One thing should be strongly emphasized, namely that smaller size of magnetic particles creates the narrower magnetic channels, and consequently, the means for disaggregating the $\mathrm{N}_{2}-\mathrm{O}_{2}-\mathrm{O}_{2}$ clusters. To make the overall picture complete let us recall the results of mathematical modelling (egs. 7-10) of experimental data and hypothesis, of "dead" thickness of magnetic membrane (c.f. Figure 6).

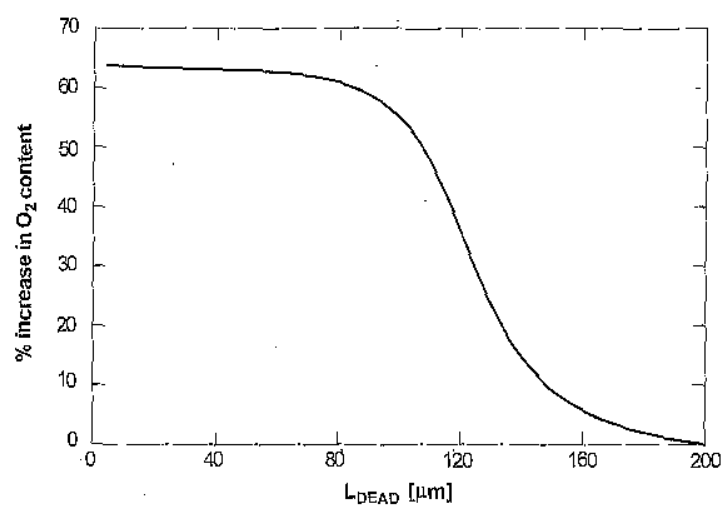

Figure 6 Percentage of oxygen concentration in permeate as a function of "dead" thickness 


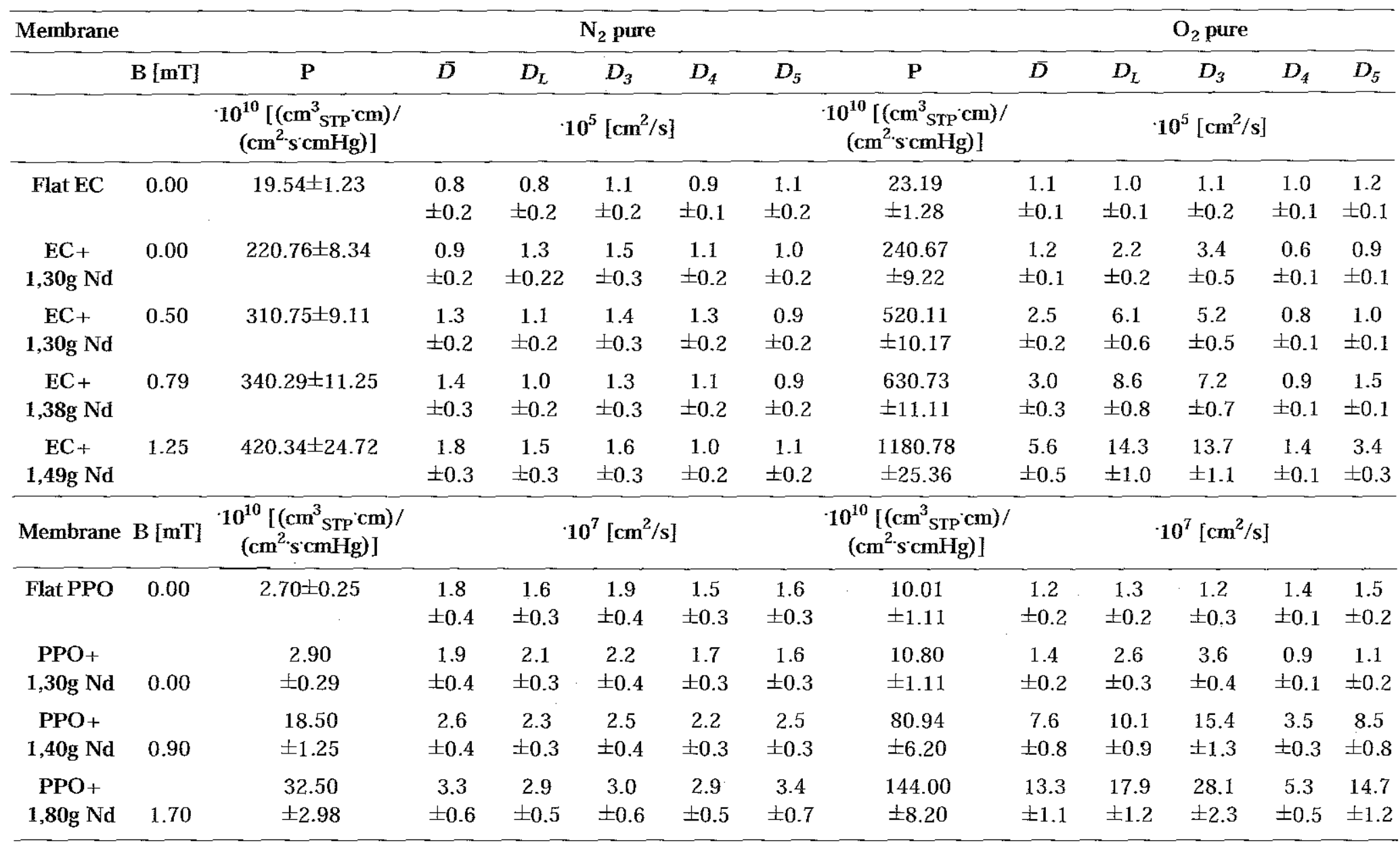


Table 2 Mass transport coefficients for nitrogen and oxygen in air

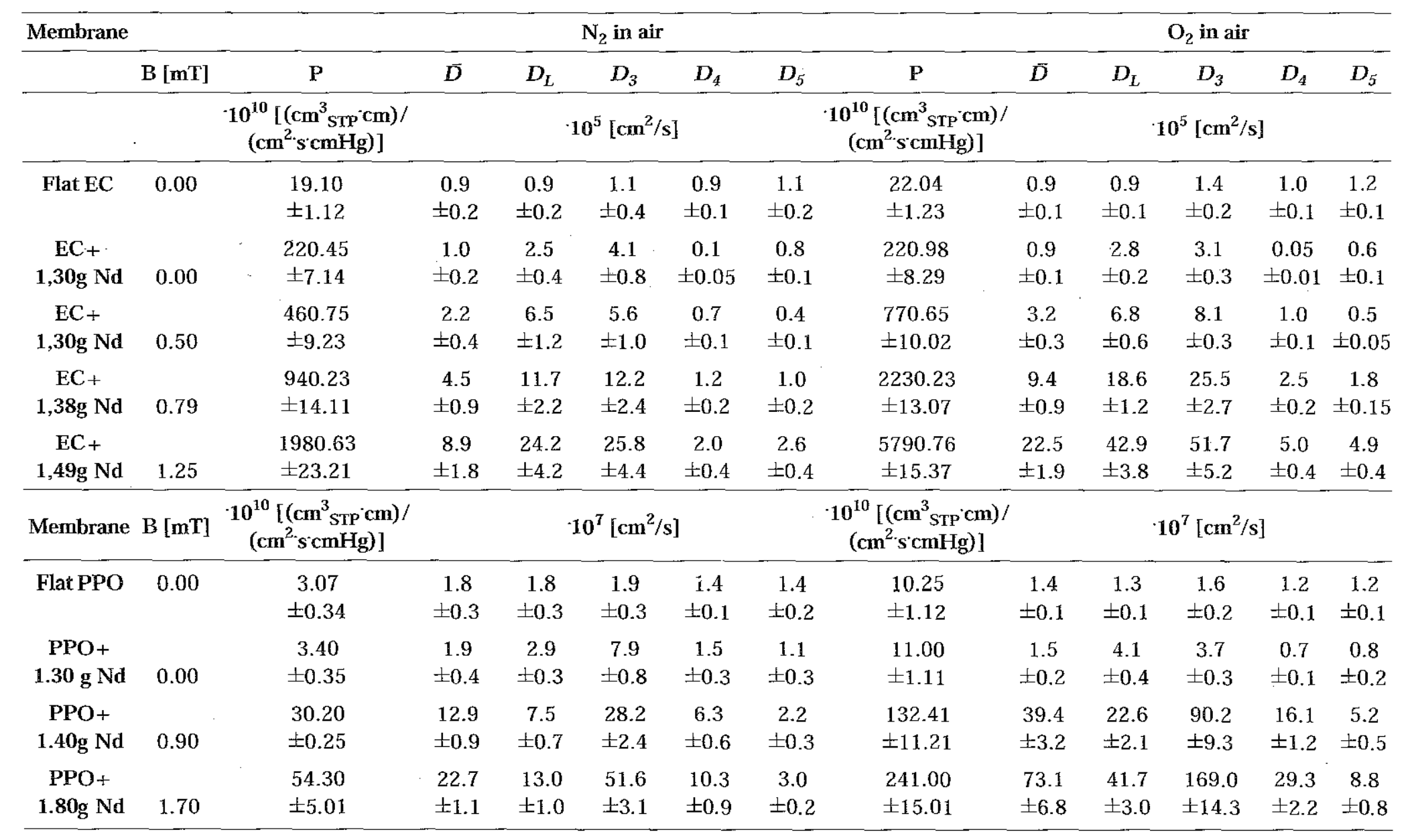


From Figure 6 one can easily see that the smaller "dead" thickness i.e. the thickness of a membrane with no magnetic particles in it, the larger percentage of the air enrichment.

The limit of about $65 \%$ is supposed to be due to the hypothesized aggregation between $\mathrm{O}_{2}$ and $\mathrm{N}_{2}$ in the ratio 2:1 [15]. There is some realistic hope that for smaller channels (fine magnetic powder), this effect (aggregation) disappears i.e. nanochannels are needed.

\subsection{CONCLUDING REMARKS}

The results presented in this paper are the "product" of three scientific tools: experiments, theory and modelling. An interplay between them produces a very interesting output in case of the air enrichment by magnetic membranes, that is, the detailed understanding of the overall process.

Consequently, we are aware of at least two crucial things that should be handled to improve our method of the air enrichment:

(i) the "dead" thickness of magnetic membrane should be cut off

(ii) the magnetic channels should be narrowed i.e. finer (as fine as possible) magnetic powder is needed.

We are in the process of managing both of the above.

\section{ACKNOWLEDGEMENTS}

This paper is dedicated to Prof. Anthony Gordon Fane with appreciation of his remarkable contribution to the membrane science. The authors would like to thank The Ministry of Science and Higher Education for providing financial support under the project (N N508 409137).

\section{REFERENCES}

[1] Strzelewicz, A. and Z. J. Grzywna. 2007. Studies on the Air Membrane Separation in the Presence of a Magnetic Field. J. Membr. Sci. 294: 60-67.

[2] Rybak, A., Z. J. Grzywna, and W. Kaszuwara. 2009. On the Air Enrichment by Polymer Magnetic Membranes. J. Membr. Sci. 336: 79-85.

[3] Rybak, A., M. Krasowska, A. Strzelewicz, and Z. J. Grzywna. 2009. Smoluchowski type Equations for Modelling of Air Separation by Membranes with Various Structure. Acta Phys. Pol. B. 40: 1447-1454.

[4] Grzywna, Z. J., A. Rybak, and A. Strzelewicz. 2010. In Membrane Gas Separation. Edited by Y. Yampolskii and B. Freeman. Wiley. 159-182.

[5] Vu, D. Q., W. J. Koros, and S. J. Miller. 2003. Mixed Matrix Membranes using Carbon Molecular Sieves. I. Preparation and Experimental Results. J. Membr. Sci. 211: 311-334.

[6] Lay, W. C. L., Y. Liu, and A. G. Fane. 2010. Impacts of Salinity on the Performance of High Retention Membrane Bioreactors for Water Reclamation: A Review. Water Research, 44: 21-40.

[7] Fane, A. G., W. Xi, and W. Rong. W. 2006 , Chapter 7: Membrane Filtration Processes and Fouling. Interface Science and Technology. 10: 109-132.

[8] Zhao, Y., Y. Tan, F.-S. Wong, A. G. Fane, and N. Xu. 2006. Formation of $\mathrm{Mg}(\mathrm{OH})_{2}$ Dynamic Membranes for Oily Water Separation: Effects of Operating conditions. Desalination. 191: 344-350.

[9] Rommelmann, D., S. Duranceau, and M. Stahl. 2004. C Kamnikar, Industrial Water Quality Requirements for Reclaimed Water. AwwaRF Report. 90979F .

[10] The EU Drinking Water Directive (DWD), Council Directive 98/83/EC.

[11] Zel'dovich, Ya. B., and Yu. P. Raizer. 1996. Physics of Shock Waves and HighTemperature Hydro-dynamic Phenomena II. New York: Academic Press.

[12] Vazquez J. L. 2006. The Porous Medium Equation. Mathematical Theory. Oxford Science Publications.

[13] Borys P., Pawelek K., and Grzywna Z. J. 2011. On the Magnetic Channels in Polymer 
membranes. Phys, Chem. Chem. Phys., 2011, 13, 17122-17129.

[14] Loeb, L. B. 1961. The Kinetic Theory of Gases. New York: Dover Publications, Inc. 278-300.
[15] Tagirov, M. S., R. M. Aminova, G. Frossati, V. N. Efimov, G. V. Mamin, V. V. Naletov, D. A. Tayurskii, and A. N. Yudin. 2009. On the Magnetism of Liquid Nitrogen-Liquid Oxygen Mixture. Physica B. 433: 329-333. 